\title{
Ganglioneuroma suprarrenal. Reporte de un caso
}

\section{Carolina Perdomo Reyes* ${ }^{\star}$ Cecilia Chambón ${ }^{\dagger}$, Daniel González González $z^{\ddagger}$}

\section{Resumen}

Introducción: el ganglioneuroma suprarrenal es un tumor benigno excepcional originado en las células nerviosas derivadas

de la cresta neural. El objetivo de este trabajo es comunicar un caso clínico de un ganglioneuroma suprarrenal derecho sintomático.

Caso clínico: paciente de 37 años, sexo femenino, que consultó por dolor abdominal inespecífico que luego de la valoración imagenológica y estudio funcional hormonal, se diagnosticó una tumoración suprarrenal derecha no funcionante. Con dicho diagnóstico se realizó la adrenalectomía por abordaje convencional retroperitoneal y el estudio anatomopatológico de la pieza diagnosticó un ganglioneuroma.

Discusión: los ganglioneuromas habitualmente son asintomáticos y no funcionantes, por lo que su diagnóstico es incidental en un alto porcentaje de casos. Otros se presentan con una sintomatología inespecíica. Su diagnóstico etiológico preoperatorio raramente es realizado y su confirmación es anatomopatológica. Tienen indicación quirúrgica los sintomáticos, los que superan los $6 \mathrm{~cm}$, o persisten dudas diagnósticas de malignidad. El pronóstico es bueno, siendo excepcional la recidiva.

Palabras clave: Ganglioneuroma

Adrenalectomía

Enfermedades de las glándulas suprarrenales

Key words: Ganglioneuroma Adrenalectomy Adrenal gland diseases

\section{Introducción}

Los ganglioneuromas son tumores benignos y poco frecuentes originados en las células del sistema nervioso simpático que habitualmente lo hacen a lo largo de toda la cadena paravertebral y la médula suprarrenal. Predominan a nivel de retroperitoneo, mediastino posterior, glándulas suprarrenales y menos frecuentemente en cuello ${ }^{(1)}$.

En las series más grandes comunicadas en la literatura, la edad media de presentación osciló entre 31 y 39 años, no siendo claro el predominio de un sexo ${ }^{(2-7)}$.

El objetivo es comunicar un caso clínico de ganglioneuroma suprarrenal derecho sintomático.

\section{Caso clínico}

Paciente de sexo femenino de 37 años que consultó por dolor en fosa ilíaca derecha de cuatro días de evolución con irradiación a fosa lumbar y sin fenómenos acompañantes. Del examen se destacó un buen estado general y dolor a la palpación de fosa ilíaca derecha, sin irritación peritoneal.

Se realizó tomografía computada que evidenció una lesión suprarrenal derecha quística, lobulada, de 97 por 59 por $87 \mathrm{~mm}$ (figura 1).

Se completó el estudio imagenológico con una resonancia magnética que no aportó nuevos datos de valor diagnóstico (figura 2).

Pruebas de función suprarrenal normales.

Con diagnóstico de tumor suprarrenal derecho sintomático se abordó por vía retroperitoneal convencional, donde se constató en la exploración una masa bien delimitada sin compromiso renal ni vena cava inferior y se realizó adrenalectomía (figura 3).

Buena evolución posterior posoperatoria. El informe del estudio anatomopatológico de la pieza concluyó que se trataba de un ganglioneuroma.

\footnotetext{
* Residente de Cirugía General.

† Asistente de Clínica Quirúrgica.

‡ Profesor titular de Clínica Quirúrgica.

Clínica Quirúrgica 3. Facultad de Medicina. Universidad de la República. Hospital Maciel. Montevideo, Uruguay.

Se consignó consentimiento del paciente para la publicación.

Los autores declaran no tener conflicto de intereses.

Correspondencia: Carolina Perdomo. Correo electrónico: carolinaperdomo09@gmail.com

Recibido: 19/11/19

Aprobado: 29/3/20
} 


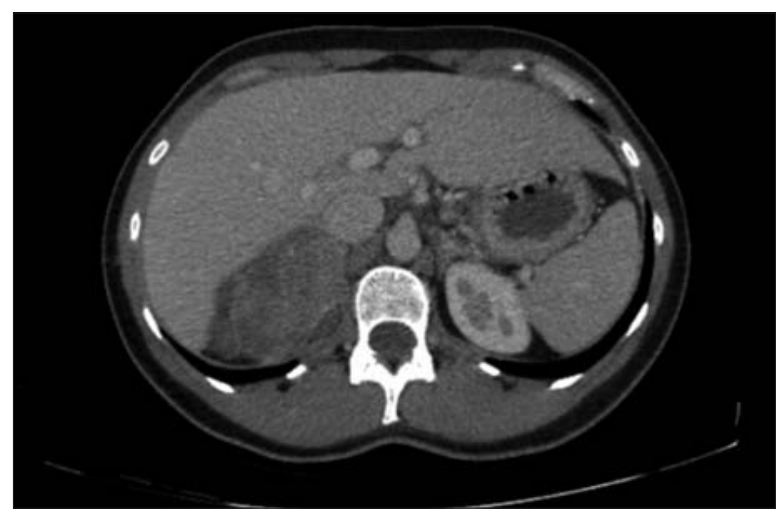

Figura 1. Tomografía computada.

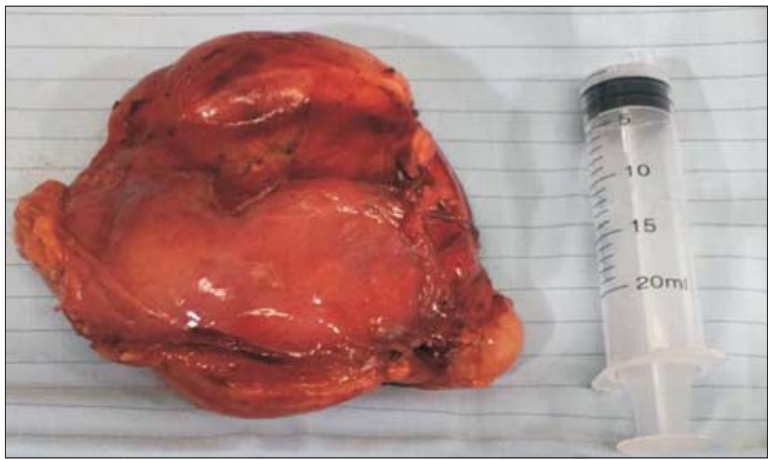

Figura 3. Pieza de resección quirúrgica.

Actualmente la paciente está en seguimiento y asintomática.

\section{Discusión}

Los ganglioneuromas originados en células ganglionares maduras, sin potencial maligno, en su gran mayoría se diagnostican bajo la forma de incidentalomas, presentándose los restantes con sintomatología vaga, generalmente disconfort abdominal o lumbar y raras veces a través de una tumoración (todos). En nuestro caso fue el dolor que sin una clara orientación etiológica determinó su evaluación mediante estudios imagenológicos y de ahí el diagnóstico de tumoración suprarrenal.

Si bien se describen algunas características tomográficas (lesión sólida, bien definida, pudiendo ser lobulada, hipodensa, encapsulada y hasta en un $40 \%$ a $60 \%$ tener calcificaciones), al igual que en la resonancia magnética (hipointensos en $\mathrm{T} 1 \mathrm{y}$ heterogéneos e hiperintensos en T2) en general no son patognomócos ${ }^{(1)}$.

El PET TC se ha empleado en aquellas tumoraciones que a pesar de haber sido estudiadas por otros métodos imagenológicos aún persisten dudas diagnósticas, fundamentalmente con la finalidad de diferenciar lesiones benignas de malignas ${ }^{(8)}$.
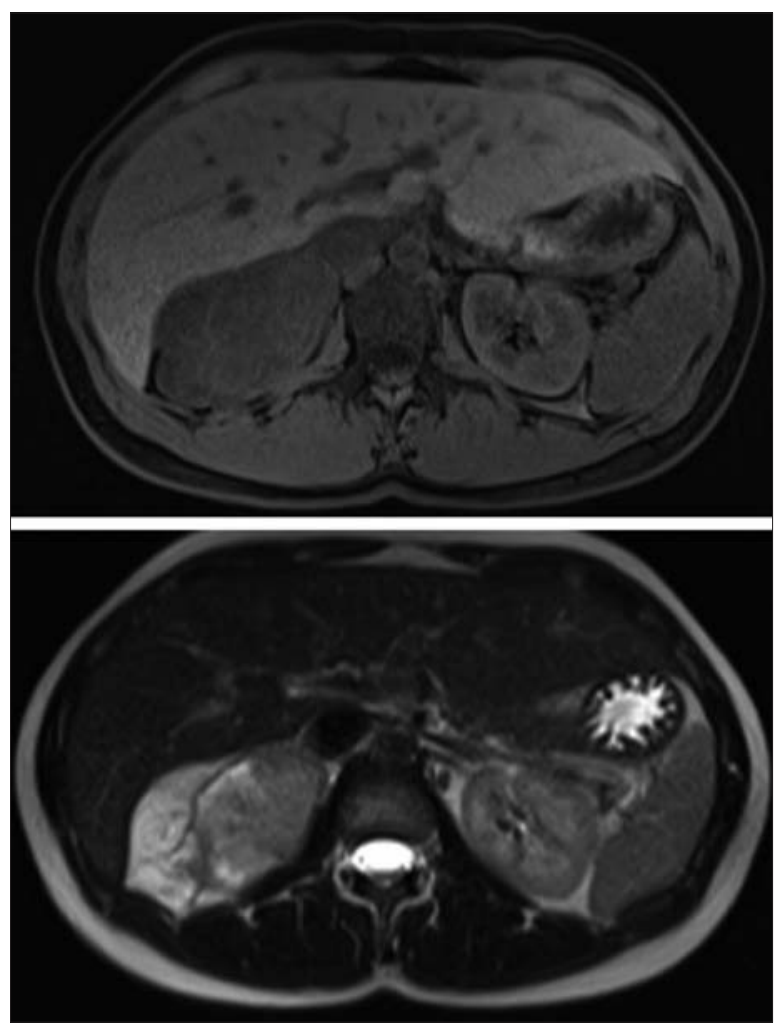

Figura 2. Resonancia magnética. Fases T1 y T2.

Otra característica de estas lesiones es que en su gran mayoría no son secretantes de hormonas ${ }^{(3,4)}$, por lo que la cirugía - de aquellas que tienen indicación quirúrgica- termina siendo el último gesto diagnóstico y el estudio anatomopatológico de la pieza el que lo establece (todos menos el último).

En tumoraciones no funcionantes la indicación quirúrgica surge cuando son sintomáticas -como el caso que presentamos- $\mathrm{o}$ asintomáticas, pero con un tamaño superior a $6 \mathrm{~cm}$ o existe la sospecha imagenológica de malignidad.

La adrenalectomía por abordaje laparoscópico (transabdominal o retroperitoneal) tiene todas las ventajas del abordaje miniinvasivo, pero tiene sus limitaciones cuando se trata de tumoraciones grandes, como el caso que presentamos. En ellos es factible la conversión en caso de haber comenzado el procedimiento por vía laparoscópica. En otros, incluyendo el presente caso, se opta por un abordaje convencional desde el inicio (todos menos el último).

El pronóstico es bueno, mejorando la sintomatología en aquellos casos que la presentaban. La recurrencia es excepcional, habiendo seguimientos desde 50 meses a 35 años ${ }^{(2,4)}$. 


\section{Summary}

Introduction: adrenal ganglioneuroma is an exceptional benign tumour which originates from neural crest cells. The study aims to inform about a clinical case of asymptomatic right adrenal ganglioneuroma.

Clinical case: 37 -year old patient, female, who consulted for non-specific abdominal pain which, after imaging assessment and functional and hormone test was diagnosed with non-functioning tumour of the right adrenal gland. Upon this diagnosis, adrenalectomy using the conventional retroperitoneal approach was performed, and the pathology study of the piece confirmed the diagnosis of ganglioneuroma.

Discussion: ganglioneuromas are usually asymptomatic and non-functioning, so diagnosis is incidental in a large percentage of cases. Others present non-specific symptoms. Preoperative etiological diagnosis is rare, and confirmation is usually after pathology study. Symptomatic tumours over $6 \mathrm{~cm}$ long have an indication of surgery, as well as those suspicious of malignancy. Prognosis is good, relapses being exceptional.

\section{Resumo}

Introdução: o ganglioneuroma suprarrenal é um tumor benigno excepcional originado nas células nervosas derivadas da crista neural.

O objetivo deste trabalho é descrever o caso clínico de um ganglioneuroma suprarrenal direito sintomático.

Caso clínico: paciente de 37 anos, sexo feminino que consultou por dor abdominal inespecífica que foi diagnosticado como uma tumoração suprarrenal direita não funcionante depois da avaliação de estudos de imagem e funcional hormonal. Com este diagnóstico realizou-se adrenalectomia por abordagem convencional retroperitoneal e o laudo anatomopatológico diagnosticou um ganglioneuroma.

Discussão: os ganglioneuromas geralmente são assintomáticos e não funcionantes por isso seu diagnóstico é acidental em uma alta proporção dos casos. Outros se apresentam com sintomatologia inespecífica. O diagnóstico etiológico pré-operatório raramente é realizado e sua confirmação é feita pela anatomia patológica. Quando são sintomáticos, têm mais de $6 \mathrm{~cm}$ ou se persistem dúvidas sobre sua malignidade têm indicação cirúrgica. O prognóstico é bom, e a recidiva é excepcional.

\section{Bibliografía}

1. Mylonas KS, Schizas D, Economopoulos KP. Adrenal ganglioneuroma: what you need to know. World J Clin Cases 2017; 5(10):373-7.

2. Fan H, Li HZ, Ji ZG, Shi BB, Zhang YS. Diagnosis and treatment of adrenal ganglioneuroma: a report of 80 cases. Zhonghua Wai Ke Za Zhi 2017; 55(12):938-41.

3. Qing Y, Bin X, Jian W, Li G, Linhui W, Bing L, et al. Adrenal ganglioneuromas: a 10-year experience in a Chinese population. Surgery 2010; 147(6):854-60.

4. Shawa H, Elsayes KM, Javadi S, Morani A, Williams MD, Lee JE, et al. Adrenal ganglioneuroma: features and outcomes of 27 cases at a referral cancer centre. Clin Endocrinol (Oxf) 2014; 80(3):342-7.

5. Spinelli C, Rossi L, Barbetta A, Ugolini C, Strambi S. Incidental ganglioneuromas: a presentation of 14 surgical cases and literature review. J Endocrinol Invest 2015; 38(5):547-54.

6. Xie J, Dai J, Zhou WL, Sun FK. Adrenal ganglioneuroma: features and outcomes of 42 cases in a chinese population. World J Surg 2018; 42(8):2469-75.

7. Li L, Shao J, Gu J, Wang X, Qu L. Adrenal ganglioneuromas: experience from a retrospective study in a Chinese population. Urol J 2014; 11(2):1485-90.

8. Mackie GC, Shulkin BL, Ribeiro RC, Worden FP, Gauger PG, Mody RJ, et al. Use of [18F] fluorodeoxyglucose positron emission tomography in evaluating locally recurrent and metastatic adrenocortical carcinoma. J Clin Endocrinol Metab 2006; 91(7):2665-71.

\footnotetext{
Contribución de autores

Los autores participaron en igual medida en todas las etapas de elaboración del artículo.

Carolina Perdomo Reyes, https://orcid.org/0000.0002.0786.1167

Cecilia Chambón, https://orcid.org/ 0000-0001-9388-8886

Daniel González González, https://orcid.org/0000.0003.3916.9201.
} 